\title{
Revisiting the application of Immobilized Artificial Membrane (IAM) chromatography to estimate in vivo distribution properties of drug discovery compounds based on the model of marketed drugs
}

\author{
Klara Valko*, Silvia Rava ${ }^{1}$, Shenaz Bunally ${ }^{2}$ and Scott Anderson ${ }^{3}$ \\ Bio-Mimetic Chromatography Ltd. Business and Technology Centre, Bessemer Drive, Stevenage, SG1 2DX, United \\ Kingdom \\ ${ }^{1}$ University of Pavia, Italy, Erasmus internship at Bio-Mimetic Chromatography Ltd. Business and Technology Centre, \\ Bessemer Drive, Stevenage, SG1 2DX, United Kingdom \\ ${ }^{2}$ Physicochemical Group, GSK, Gunnels Wood Road, Stevenage, SG1 2NY United Kingdom \\ ${ }^{3}$ Regis Technologies Inc. 8210 Austin Avenue Morton Grove, IL US 60053
}

*Corresponding Author: E-mail: Klara Valko@bio-mimetic-chromatography.com;

Received: November 27, 2019; Revised: January 06, 2020; Available online: January 31, 2020

\begin{abstract}
Immobilized Artificial Membrane (IAM) chromatography columns have been used to model the in vivo distribution of drug discovery compounds. Regis Technologies Inc., the manufacturer, had to replace the silica support and consequently introduced a new IAM.PC.DD2 column that shows slightly different selectivity towards acidic and basic compounds. The application of the new IAM.PC.DD2 columns has been evaluated and the in vivo distribution models have been compared with the previous batches of columns. It was found that due to the improved endcapping of the silica, some of the positively charged drug molecules showed shorter retention than previously published. Therefore, the column system suitability data have been updated. However, these differences do not significantly affect the previously published models for the volume of distribution, brain tissue binding and drug efficiency. Therefore, the published models can be used with the new IAM.PC.DD2 columns.
\end{abstract}

(C)2020 by the authors. This article is an open-access article distributed under the terms and conditions of the Creative Commons Attribution license (http://creativecommons.org/licenses/by/4.0/).

\section{Keywords}

Phospholipid binding, HPLC, volume of distribution, tissue binding, drug efficiency.

\section{Introduction}

Pidgeon and Venkataran [1] patented a method for immobilizing phospholipids on HPLC-grade silica stationary phases. The immobilized phospholipids mimic the lipid environment of a fluid cell membrane on a solid matrix. The retention factors $(k)$ of compounds obtained on the IAM stationary phase are proportional to their affinity (partition coefficient) $(K)$ to phospholipids according to the equation (1):

$$
\log k(\operatorname{IAM})=\log K(\operatorname{IAM})+\log \left(V_{s} / V_{m}\right)
$$

where $k$ is the retention factor obtained from the retention time $\left(t_{R}\right)$ and the dead time $\left(t_{0}\right)$ according to 
equation (2)

$$
k=\left(t_{\mathrm{R}}-t_{0}\right) / t_{0}
$$

and $V_{\mathrm{s}} / V_{\mathrm{m}}$ is the volume ratio of the stationary and mobile phases, respectively. Many drug molecules bind strongly to phospholipids as it is a pre-requisite for membrane permeability. To reduce the retention times various concentrations of acetonitrile can be used in the mobile phase. There is a linear relationship between the log $k$ values and the percentage of acetonitrile in the mobile phase that allows the extrapolation of the retention factor to the zero percentage of acetonitrile, i.e. to the pure aqueous buffer $\left(\log k_{0}\right)$ as shown by equation 3 .

$$
\log k=\text { slope } \cdot \mathrm{ACN} \%+\log k_{0}
$$

Equation 3 enables the calculation of the acetonitrile concentration that is necessary to achieve $\log k=0$, when the retention time is exactly double that of the dead time, which means an equal distribution of the compound in the mobile and the IAM stationary phase [2]. This acetonitrile concentration expressed in volume percentage is called the Chromatographic Hydrophobicity Index (CHI (IAM)) [3]. It has also been shown that the CHI (IAM) values have linear relationships with the gradient retention times of the compounds obtained on the IAM stationary phases. By using an acetonitrile gradient of $0 \%$ to a maximum of $85 \%$ the obtained gradient retention times can be calibrated from the CHI (IAM) values by using a set of standards, eliminating the need to carry out isocratic measurements. The so obtained CHI (IAM) values show a good correlation to the $\log k_{0}$ (IAM) values, that are the extrapolated logarithmic retention factors to $0 \%$ acetonitrile concentration in the isocratic mode. The chromatographic determination of phospholipid-binding is based on measuring gradient retention times. It is independent of the amount of compound injected onto the column, so there is no need for quantitative analysis. The time measurement is very reproducible, and the calibrated (relative) gradient retention data are suitable for inter-laboratory comparison and application in published models of in vivo drug distribution. The data so obtained can be compiled in databases and is suitable for use in establishing quantitative structureretention relationships, thus enabling chemists to design compounds with the appropriate IAM binding.

The application of IAM chromatography as an aid in drug discovery has been reviewed extensively in recent years [4-8]. The molecular factors that influence IAM retention have also been discussed [9,10]. It has been found that IAM columns retain strongly positively charged compounds as the IAM columns are negatively charged on the surface similar to cell membranes [11-13]. Several applications of IAM chromatography have also published [14] and this is summarized below.

Intestinal absorption and drug distribution depend on a compound's partitioning into phospholipids. Compounds have to possess a certain degree of affinity to membranes in order to permeate through the biological phospholipid bilayers. Therefore, IAM chromatography can provide an insight into the potential intestinal absorption of compounds [15]. As tissues represent a more non-polar lipid environment relative to the plasma, the human volume of distribution can be modelled by the binding differences between IAM and human serum albumin (HSA) $[13,16]$, as shown by equation (4):

$$
\log V_{\mathrm{dss}}=0.44 \log K(\mathrm{IAM})-0.22 \log K(\mathrm{HSA})-0.66
$$

The sum of the albumin and phospholipid-binding inversely correlates to the unbound fraction of the compounds in tissues according to equation (5) [17].

$$
\log f_{\mathrm{ut}}=-0.66 \log K(\mathrm{HSA})-0.52 \log K(\mathrm{IAM})+0.55
$$


The in vivo drug efficiency can be modelled by the sum of the IAM and HSA binding of compounds [18] according to equation 6. The in vitro $\mathrm{DRUG}_{\text {eff }}$ shows an excellent correlation with the in vivo $\mathrm{DRUG}_{\text {eff. }}$ Drug efficiency and potency enables early dose estimation as drug efficiency approximates to the proportion of free drug concentration and dose [19].

$\log D_{R U G}=2-(0.23 \log K(\mathrm{HSA})+0.43 \log K($ IAM) -0.72$)$

It has been found that basic compounds that have $\mathrm{CHI}$ (IAM) value greater than 50 have phospholipidosis potential [20] and also show promiscuous binding, interacting with several targets, causing potential toxicity and side effects [21].

At present Regis Technologies Inc. (Morton Grove, IL, USA) is the only manufacturer of the Immobilized Artificial Membrane (IAM) stationary phase. The stationary phase contains only a monolayer of the phosphatidylcholine (PC) with the aliphatic part chemically bonded to the silica while the choline polar head group faces towards the mobile phase. Initially, "Type A" silica was used in the bonding procedure. The PC molecule has only one alkyl chain available with the second alkyl chain directly bonded to the silica surface. In this way, a denser bonding could be achieved which was closer to the natural density of PC molecules on the cell surface. However, in 2018 the company had to change the base silica material to a newer "Type B" as the old supply was no longer available. "Type B" silica has been chosen as the closest in properties to "Type $A$ " regarding the specific surface area, pore size and particle size. When testing the retention selectivity of the new IAM.PC.DD2 columns which were otherwise manufactured in the same way, differences were observed towards acidic and basic molecules. This was somewhat surprising as the new silica support had identical physical specifications to the previously used silica (10 $\mu \mathrm{m}$ particle size, $300 \AA$ p pore size and nearly identical surface area). However, given the newer silica is of a much higher purity with only single ppm metal content, these observations are understandable as the new silica provides a much more inert surface and likely contributes to less non-specific binding interactions.

The difference in selectivity can also be attributed to the different arrangements of the positive and negative charges of the choline headgroup on the stationary phase surface, again somewhat affected by the higher purity silica during the bonding process. The new batches of columns did not pass the original system suitability test criteria. Hence the aim was to establish new $\mathrm{CHI}$ (IAM) values for the system suitability test compounds that could be reproduced on the new batches of IAM columns. In this paper, the investigation of the selectivity difference is reported using over 70 marketed drug molecules and the in vivo distribution models are compared for the old and the new batches of IAM.PC.DD2 stationary phases. The estimated volume of distribution data was compared with the data from the previously published models. The differences of the models for the estimated brain tissue binding and drug efficiency values of the known drug molecules are investigated and compared with the published data obtained using the previous batches of IAM.PC.DD2 columns.

\section{Experimental}

All high-performance liquid chromatography measurements were performed on an Agilent 1100 Series HPLC instrument equipped with an ultraviolet diode array detector (DAD).

Measurements of membrane binding using immobilized artificial membrane (IAM) chromatography

The IAM binding was measured using IAM.PC.DD2 HPLC columns (Regis Technologies, Inc., IL, USA) with dimensions of $100 \times 4.6 \mathrm{~mm}$, particle size $10 \mu \mathrm{m}$ with $300 \AA ̊$ pore size.

Two batches of IAM.PC.DD2 columns were studied (R20511-014-3 and P20511-014-3) that had been 
prepared using the new batches of silica support. The selectivity and the models obtained were compared with the published average IAM data obtained on the columns manufactured between 2004 and 2012 [14].

Mobile phase A was 50 mM ammonium acetate adjusted to $\mathrm{pH} 7.4$ and mobile phase B was $100 \%$ acetonitrile. The mobile phase flow rate was $1.5 \mathrm{~mL} / \mathrm{min}$ and the run time was $6 \mathrm{~min}$. The acetonitrile gradient was applied from 0 to $85 \%$ from 0 to $4.75 \mathrm{~min}$ and kept at $85 \%$ until $5.15 \mathrm{~min}$. From 5.15 to $5.25 \mathrm{~min}$ the acetonitrile concentration dropped back to $0 \%$.

The retention times were standardized using the IAM calibration mixture, containing octanophenone, heptanophenone, hexanophenone, valerophenone, butyrophenone, propiophenone, acetophenone, acetanilide and paracetamol. The retention times of the compounds were plotted against the isocratically determined and predefined CHI (IAM) values [4] listed in Table 1. The slope and intercept of the obtained straight line was used to calculate the $\mathrm{CHI}$ IAM values of new compounds. The $\mathrm{CHI}$ IAM values were converted to the octanol/water lipophilicity scale and expressed as log $K$ (IAM) values using equation (7).

$$
\log K(\mathrm{IAM})=0.29 \exp (0.026 \mathrm{CHI}(\mathrm{IAM})+0.70
$$

The so-obtained log $K_{\mathrm{IAM}}$ values express the membrane partition comparable to the octanol/water lipophilicity as described in reference [22]. A typical chromatogram is shown in Figure 1.

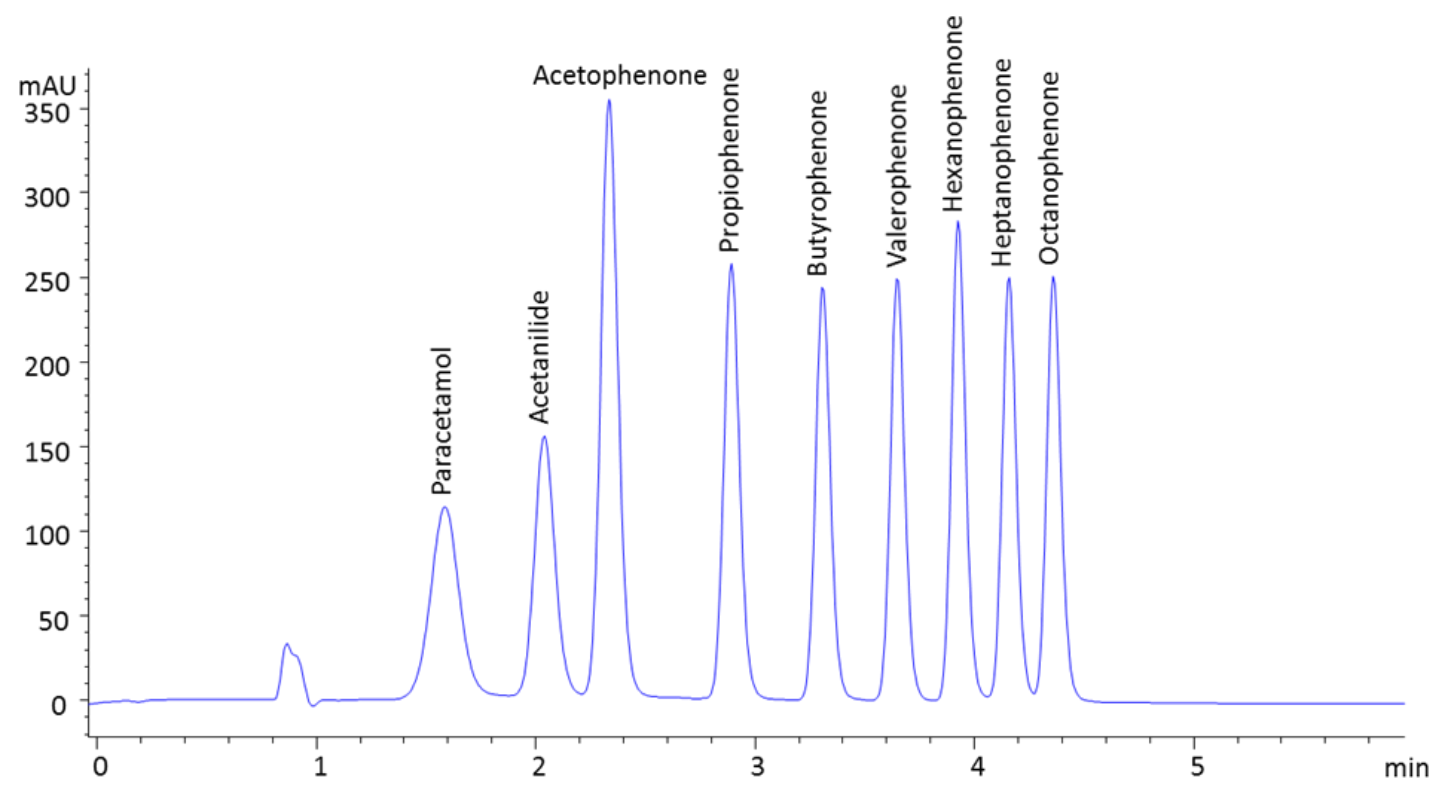

Figure 1. A typical chromatogram obtained on IAM.PC.DD2 $100 \times 4.6 \mathrm{~mm}$ HPLC column. Flow rate: $1.5 \mathrm{~mL} / \mathrm{min}$;

Mobile phase A: $50 \mathrm{mM}$ ammonium acetate adjusted to $\mathrm{pH} 7.4$, mobile phaseB: acetonitrile. Run time 6 min; Gradient: 0 to $4.75 \mathrm{~min} 0$ to $85 \%$ B, 4.75 to $5.15 \mathrm{~min} 85 \%$ B, 5.15 to $5.25 \mathrm{~min} 0 \%$ B; Detection wavelength: $250 \mathrm{~nm}$

The natural state of the phosphatidylcholine headgroup on the IAM stationary phase provides the column selectivity that was tested using the system suitability test compounds containing acidic, basic and neutral compounds.

Protein binding measurements using biomimetic protein stationary phases

The interaction of the compounds listed in Table 2 with human serum albumin (HSA) has been measured using commercially available chemically bonded HSA columns (Chiralpak-HSA from HiChrom, UK) with the dimensions of $50 \times 3 \mathrm{~mm}$ and a particle size of $5 \mu \mathrm{M}$.

Mobile phase A was $50 \mathrm{mM}$ ammonium acetate adjusted to $\mathrm{pH} 7.4$ and mobile phase $\mathrm{B}$ was 2-propanol. The flow rate was $1.5 \mathrm{~mL} / \mathrm{min}$ and the run time was $6 \mathrm{~min}$. The 2-propanol gradient was applied from 0 to 
$35 \%$ from 0 to $3.00 \mathrm{~min}$ and kept at $35 \%$ until $4.00 \mathrm{~min}$. From 4.00 to $4.25 \mathrm{~min}$ the 2-propanol concentration was dropped back to $0 \%$. Although column manufacturers suggest using $\mathrm{pH} 7$ for chiral separations using the HSA column, in this case, the $\mathrm{pH} 7.4$ mobile phase is used to mimic the plasma $\mathrm{pH}$ for the plasma protein binding measurements.

Table 1. The constant CHI (IAM) and log $K$ (IAM) values for the calibration set of compounds with typical retention times.

\begin{tabular}{lccc}
\hline Compound & CHI (IAM) & log $\boldsymbol{K}($ IAM) & $\boldsymbol{t}_{\mathrm{R}} \mathbf{7 . 4}(\mathbf{m i n})$ \\
\hline Octanophenone & 49.4 & 4.97 & 4.37 \\
Heptanophenone & 45.7 & 4.30 & 4.17 \\
Hexanophenone & 41.8 & 3.71 & 3.93 \\
Valerophenone & 37.3 & 3.16 & 3.66 \\
Butyrophenone & 32.0 & 2.62 & 3.32 \\
Propiophenone & 25.9 & 2.15 & 2.90 \\
Acetophenone & 17.2 & 1.67 & 2.34 \\
Acetanilide & 11.5 & 1.45 & 2.04 \\
Paracetamol & 2.90 & 1.20 & 1.58 \\
\hline
\end{tabular}

The retention times were standardized using the calibration set of compounds described previously [23].

\section{Compounds studied}

In this study, 72 commercial drugs from diverse therapeutic areas have been analysed. $50 \mu \mathrm{l}$ of $10 \mathrm{mM}$ DMSO solutions of the drugs were diluted down to $150 \mu \mathrm{l}$ and 10

$\mu \mathrm{l}$ of the diluted solution was injected onto the HPLC columns. The clinical volume of distribution data for all of the drugs is available in the literature [13].

Table 2 shows the marketed drug molecules and their clinical volume of distribution data obtained from reference [13] and the previously measured CHI (IAM) data [14] using an IAM column manufactured by Regis Technologies between 2004 and 2012.

\section{Data analysis}

The statistical analysis was performed using JMP 13 (SAS Institute, USA).

\section{Results and Discussion}

The selectivity and reproducibility of IAM.PC.DD2 columns have been extensively studied. First, the $\mathrm{CHI}$ (IAM) values of the system suitability test compounds (see Table 2) were scrutinized. The CHI (IAM) values of the test compounds were then compared with the $\mathrm{CHI}$ (IAM) values obtained on the new batches of IAM.PC.DD2 columns. It was found, as shown in Table 3, that carbamazepine, warfarin, indomethacin, nicardipine, ketoprofen, haloperidol and budesonide had very similar CHI (IAM) values. However, imipramine and chlorpromazine showed greater than the $5 \mathrm{CHI}$ (IAM) unit deviations which would be within the experimental error and therefore they did not pass the system suitability criteria. These compounds are strong bases and positively charged at $\mathrm{pH}$ 7.4. Most probably the new IAM.PC.DD2 columns have less negative charge on the surface and therefore reduce the retention times of positively charged compounds. It is also possible that more of the free silanol groups were end-capped which would contribute to a shorter retention of basic compounds. Therefore, the system suitability criteria were changed for basic compounds. The "new" CHI (IAM) values are listed in Table 3.

The in vivo distribution models were re-examined using the data from the new IAM.PC.DD2 columns. Because of the selectivity differences, it was essential to investigate the CHI (IAM) values for a larger set of compounds and check how the previously described in vivo distribution models performed using the data from the new columns manufactured with the new silica material.

The 72 drug molecules from the original set of marketed drugs for which the models were developed [13] were analysed on the new IAM.PC.DD2 columns and the HSA column. Table 4 shows the CHI (IAM) values and the log $k$ HSA binding data obtained from reference [13] and remeasured in this study. 
Table 2. The name of the marketed drugs, their CAS number, their previously published CHI (IAM) data and the logarithm of their clinical volume of distribution.

\begin{tabular}{|c|c|c|c|c|}
\hline Compound & CAS NO & Acid/base character & $\begin{array}{c}\mathrm{CHI} \text { (IAM) } \\
\text { [14] }\end{array}$ & $\log V_{d s s}[13]$ \\
\hline Acecainide & $32795-44-1$ & basic & 23.3 & 0.10 \\
\hline Acetanilide & $103-84-4$ & neutral & 10.7 & -0.13 \\
\hline Acetazolamide & $59-66-5$ & neutral & 1.7 & -0.46 \\
\hline Alclofenac & 22131-79-9 & acidic & 17.8 & -1.10 \\
\hline Amoxapine & $14028-44-5$ & basic & 56.5 & 1.67 \\
\hline Bamethan & $3703-79-5$ & basic & 18.6 & 0.01 \\
\hline Betamethasone & $378-44-9$ & neutral & 31.7 & 0.18 \\
\hline Carbamazepine & $298-46-4$ & neutral & 26.5 & -0.09 \\
\hline Chlorpromazine & $50-53-3$ & basic & 61.9 & 2.15 \\
\hline Cinoxacin & $28657-80-9$ & acidic & 1.6 & -0.42 \\
\hline Colchicine & $64-86-8$ & neutral & 23.7 & 0.03 \\
\hline Cytarabine & $147-94-4$ & weak base & -15.0 & -0.31 \\
\hline Diazoxide & $364-98-7$ & weak acid & 24.7 & -0.16 \\
\hline Diclofenac & $15307-86-5$ & acidic & 33.5 & -0.67 \\
\hline Diprophylline & $479-18-5$ & neutral & -4.0 & -0.24 \\
\hline Ethinyl estradiol & $57-63-6$ & neutral & 46.8 & 0.39 \\
\hline Famotidine & $76824-35-6$ & weak base & 15.7 & -0.06 \\
\hline Felbamate & $25451-15-4$ & neutral & 19.1 & -0.64 \\
\hline Felodipine & $72509-76-3$ & neutral & 46.1 & 0.35 \\
\hline Fenoprofen & $31879-05-7$ & acidic & 17.2 & -1.27 \\
\hline Finasteride & $98319-26-7$ & neutral & 38.9 & 0.30 \\
\hline Floxacillin & $5250-39-5$ & acidic & 23.4 & -0.56 \\
\hline Flumazenil & $78755-81-4$ & neutral & 18.4 & -0.04 \\
\hline Flurbiprofen & $5104-49-4$ & acidic & 26.8 & -1.56 \\
\hline Furosemide & $54-31-9$ & acidic & 21.4 & -0.71 \\
\hline Gemfibrozil & $25812-30-0$ & acidic & 32.9 & -1.34 \\
\hline Glipizide & $29094-61-9$ & acidic & 21.1 & -0.73 \\
\hline Griseofulvin & $126-07-8$ & neutral & 33.1 & 0.12 \\
\hline Hydrochlorothiazide & $58-93-5$ & weak acid & 15.9 & -0.13 \\
\hline Hydrocortisone & $50-23-7$ & neutral & 27.9 & 0.12 \\
\hline Imipramine & $50-49-7$ & basic & 51.6 & 1.16 \\
\hline Indomethacin & $53-86-1$ & acidic & 25.3 & -0.96 \\
\hline Isradipine & $75695-93-1$ & neutral & 40.0 & 0.13 \\
\hline Ketoconazole & $65277-42-1$ & weak base & 42.9 & 0.32 \\
\hline Ketoprofen & $22071-15-4$ & acidic & 21.9 & -1.01 \\
\hline Labetalol & $36894-69-6$ & amphoteric & 43.9 & 0.71 \\
\hline Lignocaine & $137-58-6$ & basic & 32.6 & 0.40 \\
\hline Methylprednisolone & $83-43-2$ & neutral & 32.1 & 0.16 \\
\hline Metronidazole & $443-48-1$ & weak base & -3.3 & -0.25 \\
\hline Minoxidil & $38304-91-5$ & weak base & 19.0 & -0.04 \\
\hline Nabumetone & $42924-53-8$ & neutral & 38.4 & 0.03 \\
\hline Nadolol & $42200-33-9$ & basic & 20.2 & -0.33 \\
\hline Nicardipine & $55985-32-5$ & weak base & 45.9 & 0.47 \\
\hline Nifedipine & $21829-25-4$ & neutral & 29.0 & -0.04 \\
\hline Nitrendipine & $39562-70-4$ & neutral & 40.5 & 0.16 \\
\hline
\end{tabular}




\begin{tabular}{lcccc}
\hline Papaverine & $58-74-2$ & weak base & 34.4 & 0.00 \\
Pentoxifylline & $6493-05-6$ & neutral & 12.0 & -0.10 \\
Perphenazine & $58-39-9$ & basic & 56.3 & 1.35 \\
Phenytoin & $57-41-0$ & weak acid & 31.6 & 0.03 \\
Pindolol & $13523-86-9$ & basic & 42.0 & 0.85 \\
Prazosin & $19216-56-9$ & weak base & 31.6 & -0.05 \\
Prednisolone & $50-24-8$ & neutral & 28.0 & 0.14 \\
Prednisone & $53-03-2$ & neutral & 25.9 & 0.05 \\
Primidone & $125-33-7$ & neutral & 8.9 & -0.15 \\
Probenecid & $57-66-9$ & acidic & 20.1 & -0.75 \\
Procainamide & $614-39-1$ & basic & 19.9 & 0.02 \\
Propanolol & $525-66-6$ & basic & 50.8 & 1.29 \\
Propylthiouracil & $51-52-5$ & weak acid & 3.9 & -0.34 \\
Proxyphylline & $603-00-9$ & neutral & 1.1 & -0.85 \\
Sulfachlorpyridazine & $80-32-0$ & acidic & 6.0 & -0.76 \\
Sulfameter & $651-06-9$ & acidic & 3.7 & -0.61 \\
Sulfamethoxypyridazine & $80-35-3$ & weak acid & 9.6 & -0.59 \\
Sulfinpyrazone & $57-96-5$ & acidic & 26.1 & -0.65 \\
Sulfisoxazole & $127-69-5$ & acidic & 2.9 & -0.72 \\
Sulphadimidine & $57-68-1$ & amphoteric & 34.6 & 0.22 \\
Sulpiride & $15676-16-1$ & basic & 25.5 & 0.12 \\
Tamoxifen & $10540-29-1$ & basic & 58.7 & 1.48 \\
Theobromine & $83-67-0$ & weak acid & -4.1 & -0.25 \\
Tolfenamic Acid & $13710-19-5$ & acidic & 36.6 & -0.76 \\
Trazodone & $19794-93-5$ & weak base & 36.3 & 0.01 \\
Trimethoprim & $738-70-5$ & weak base & 20.8 & -0.06 \\
Warfarin & $81-81-2$ & acidic & 19.9 & -0.78 \\
\hline & & & & \\
\hline
\end{tabular}

Table 3. The $\mathrm{CHI}$ (IAM) obtained from the literature [13] (CHI (IAM) reference), the $\mathrm{CHI}$ (IAM) values obtained on the new IAM.PC.DD2 columns and the acid/base character of the IAM calibration compounds

\begin{tabular}{cccc}
\hline Compound & Acid/base character & CHI (IAM) & CHI (IAM) “new" \\
\hline Carbamazepine & neutral & 28.9 & 27.0 \\
Colchicine & neutral & 23.7 & 23.0 \\
Warfarin & acidic & 19.9 & 24.0 \\
Indomethacin & acidic & 32.5 & 30.0 \\
Nicardipine & weak base & 45.9 & 45.0 \\
Propranolol & basic & 45.1 & 42.0 \\
Imipramine & basic & 54.1 & 45.0 \\
Ketoprofen & strong acid & 21.9 & 20.0 \\
Chlorpromazine & basic & 61.9 & 54.0 \\
Haloperidol & basic & 44.3 & 48 \\
Budesonide & neutral & 38.7 & 38 \\
\hline
\end{tabular}

Figure 2 shows the plot of the experimental CHI (IAM) values on IAM.PC.DD2 columns P20511-014-3 and R2055-014-3. The CHI (IAM) values obtained on the columns are very similar indicating good batch to batch reproducibility of the new columns. 
Table 4. The experimental CHI (IAM) values of the investigated compounds obtained on the new IAM.PC.DD2 columns and the CHI (IAM) obtained from the literature [13] (reference CHI (IAM))

\begin{tabular}{|c|c|c|c|c|c|}
\hline Compound & $\begin{array}{c}\text { CHI (IAM) } \\
\text { from ref [13] }\end{array}$ & $\begin{array}{l}\text { R20511-014-3 } \\
\text { CHI (IAM) }\end{array}$ & $\begin{array}{l}\text { P20511-014-3 CHI } \\
\text { (IAM) }\end{array}$ & $\begin{array}{l}\log K \text { HSA } \\
\text { from ref [13] }\end{array}$ & $\log K \mathrm{HSA}$ \\
\hline Acecainide & 23.30 & 17.72 & 17.57 & 0.69 & 0.52 \\
\hline Acetanilide & 10.70 & 11.55 & 11.50 & 0.62 & 0.43 \\
\hline Acetazolamide & 1.66 & 0.99 & 0.74 & 1.47 & 1.44 \\
\hline Alclofenac & 17.80 & 20.47 & 21.63 & 5.11 & 5.40 \\
\hline Amoxapine & 56.50 & 45.41 & 44.74 & 2.15 & 2.70 \\
\hline Bamethan & 18.60 & 18.53 & 18.03 & 0.49 & 0.42 \\
\hline Betamethasone & 31.70 & 30.18 & 30.05 & 1.09 & 1.39 \\
\hline Carbamazepine & 26.50 & 27.18 & 27.07 & 1.99 & 1.81 \\
\hline Chlorpromazine & 61.90 & 51.54 & 50.94 & 1.18 & 1.27 \\
\hline Cinoxacin & 1.60 & 5.31 & 6.67 & 0.79 & 0.91 \\
\hline Colchicine & 23.70 & 23.45 & 23.18 & 0.69 & 0.24 \\
\hline Cytarabine & -15.00 & -6.76 & -6.99 & 1.59 & 1.87 \\
\hline Diazoxide & 24.70 & 23.39 & 23.44 & 5.42 & 5.58 \\
\hline Diclofenac & 33.50 & 35.18 & 35.85 & 0.63 & 0.24 \\
\hline Diprophylline & -4.00 & -1.49 & -1.98 & 4.1 & 4.24 \\
\hline Ethinyl Estradiol & 46.80 & 45.30 & 45.23 & 0.46 & 0.50 \\
\hline Famotidine & 15.70 & 16.82 & 16.08 & 1.39 & 3.43 \\
\hline Felbamate & 19.07 & 39.16 & 39.21 & 3.46 & 4.15 \\
\hline Felodipine & 46.10 & 44.37 & 44.45 & 5.91 & 6.11 \\
\hline Fenoprofen & 17.20 & 27.17 & 28.26 & 1.58 & 2.32 \\
\hline Finasteride & 38.90 & 37.15 & 37.17 & 3.62 & 3.55 \\
\hline Floxacillin & 23.40 & 23.76 & 24.59 & 0.56 & 0.65 \\
\hline Flumazenil & 18.40 & 18.27 & 18.27 & 7.81 & 8.50 \\
\hline Flurbiprofen & 26.80 & 30.23 & 30.89 & 1.26 & 4.01 \\
\hline Furosemide & 21.41 & 21.96 & 22.69 & 3.64 & 8.50 \\
\hline Gemfibrozil & 32.90 & 33.70 & 34.53 & 3.32 & 4.05 \\
\hline Glipizide & 21.10 & 22.82 & 23.22 & 1.49 & 1.92 \\
\hline Griseofulvin & 33.10 & 31.06 & 30.98 & 0.77 & 0.80 \\
\hline Hydrochlorothiazide & 15.90 & 13.04 & 13.08 & 0.92 & 1.05 \\
\hline Hydrocortisone & 27.90 & 27.23 & 27.02 & 1.95 & 2.59 \\
\hline Imipramine & 51.60 & 45.58 & 44.81 & 6.17 & 5.59 \\
\hline Indomethacin & 25.30 & 32.49 & 33.16 & 2.86 & 3.36 \\
\hline Isradipine & 40.00 & 39.11 & 39.16 & 2.9 & 3.30 \\
\hline Ketoconazole & 42.90 & 39.50 & 39.26 & 4.8 & 5.40 \\
\hline Ketoprofen & 21.90 & 22.93 & 23.89 & 1.28 & 1.80 \\
\hline Labetalol & 43.90 & 38.29 & 37.48 & 0.69 & 0.52 \\
\hline Lignocaine & 32.60 & 24.89 & 25.53 & 1.35 & 1.53 \\
\hline Methylprednisolone & 32.10 & 30.91 & 30.77 & 0.76 & 0.28 \\
\hline Metronidazole & -3.30 & -2.71 & -3.24 & 0.66 & 0.72 \\
\hline Minoxidil & 19.00 & 15.93 & 16.08 & 3.16 & 3.41 \\
\hline Nabumetone & 38.40 & 35.90 & 35.96 & 0.49 & 2.14 \\
\hline Nadolol & 20.20 & 19.89 & 19.23 & 2.93 & 3.55 \\
\hline Nicardipine & 45.90 & 43.82 & 43.73 & 1.41 & 1.95 \\
\hline Nifedipine & 29.00 & 28.11 & 28.16 & 3.13 & 3.37 \\
\hline
\end{tabular}




\begin{tabular}{|c|c|c|c|c|c|}
\hline Nitrendipine & 40.50 & 39.06 & 39.19 & 2.35 & 2.70 \\
\hline Papaverine & 34.40 & 31.22 & 31.15 & 0.63 & 0.39 \\
\hline Pentoxifylline & 12.00 & 14.04 & 13.87 & 3.27 & 4.03 \\
\hline Perphenazine & 56.30 & 49.57 & 48.97 & 1.6 & 2.02 \\
\hline Phenytoin & 31.60 & 30.18 & 30.20 & 0.54 & 0.63 \\
\hline Pindolol & 42.00 & 25.69 & 25.31 & 1.81 & 2.40 \\
\hline Prazosin & 31.60 & 29.82 & 29.35 & 0.89 & 0.95 \\
\hline Prednisolone & 28.00 & 27.31 & 27.20 & 0.8 & 1.08 \\
\hline Prednisone & 25.90 & 25.25 & 25.13 & 0.62 & 0.42 \\
\hline Primidone & 8.90 & 10.31 & 10.49 & 3.44 & 4.01 \\
\hline Probenecid & 20.10 & 23.45 & 24.38 & 0.76 & 0.49 \\
\hline Procainamide & 19.90 & 11.73 & 11.89 & 0.73 & 1.67 \\
\hline Propranolol & 50.80 & 42.24 & 41.46 & 0.63 & 1.02 \\
\hline Propylthiouracil & 3.90 & 6.56 & 6.52 & 2.67 & 3.21 \\
\hline Proxyphylline & 1.10 & 10.05 & 10.89 & 1.97 & 3.02 \\
\hline Sulfachlorpyridazine & 6.00 & 10.08 & 10.83 & 2.04 & 2.20 \\
\hline Sulfameter & 3.70 & 6.92 & 7.09 & 4.09 & 2.44 \\
\hline Sulfamethoxypyridazine & 9.60 & 11.11 & 11.16 & 2.33 & 4.27 \\
\hline Sulfinpyrazone & 26.10 & 26.19 & 26.89 & 3.17 & 2.66 \\
\hline Sulfisoxazole & 2.90 & 6.48 & 7.37 & 1.54 & 3.68 \\
\hline Sulphadimidine & 34.55 & 10.89 & 11.09 & 0.75 & 1.72 \\
\hline Sulpiride & 25.50 & 15.80 & 16.15 & 4.84 & 0.71 \\
\hline Tamoxifen & 58.70 & 54.23 & 53.78 & 1.29 & 4.82 \\
\hline Theobromine & -4.10 & -3.16 & -3.71 & 4.68 & 0.27 \\
\hline Tolfenamic Acid & 36.60 & 40.72 & 41.53 & 2.46 & 6.60 \\
\hline Trazodone & 36.30 & 34.09 & 33.86 & 0.8 & 3.06 \\
\hline Trimethoprim & 20.80 & 20.99 & 20.52 & 4.45 & 0.95 \\
\hline Warfarin & 19.90 & 23.47 & 24.18 & 0.7 & 4.16 \\
\hline
\end{tabular}

Figure 3 shows the plot of the $\mathrm{CHI}$ (IAM) from the literature [13], reference $\mathrm{CHI}$ (IAM), with the experimental CHI (IAM) values using the IAM.PC.DD2 column R2055-014-3. The CHI (IAM) values of procainamide, sulpiride, zolmitriptan and pindolol were outliers from the correlation with lower CHI (IAM) values on the new batches of IAM columns. Compounds are considered outliers when the difference between the measured and predicted value is greater than the double of the standard error of the estimate, in this case 7.2 CHI IAM units. The slope of the regression line (red) is less than one (0.81) and the regression line is different from the line of unity (green). When these 4 strong basic compounds are left out from the correlation, the correlation coefficient increases to 0.96 and the slope increases to 0.82 as shown in Figure 4.

The results shown in Figure 4 suggests that the rank order of the phospholipid binding will be very similar using the CHI IAM data obtained on the new IAM.PC.DD2 column and the old IAM.PC.DD2 column. However, some basic compounds may show significantly weaker phospholipid binding. The absolute $\mathrm{CHI}$ IAM values maybe lower when the new batches of IAM columns are used. 


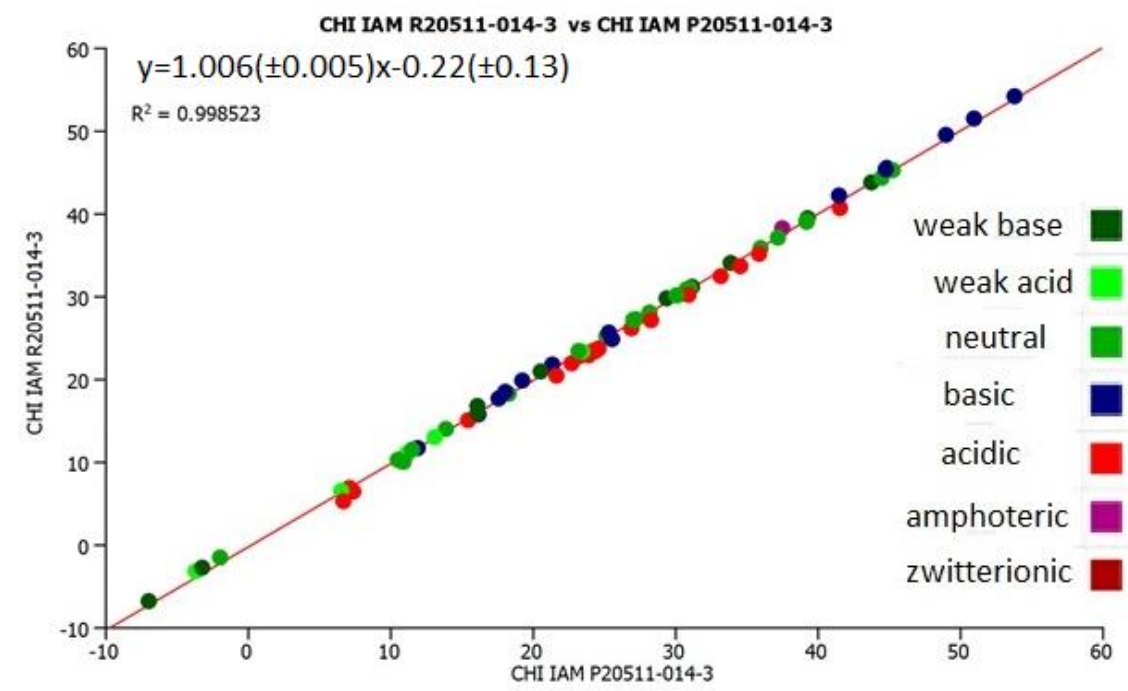

Figure 2. The plot of the experimental CHI (IAM) values on IAM.PC.DD2 columns P20511-014-3 and R20511-014-3

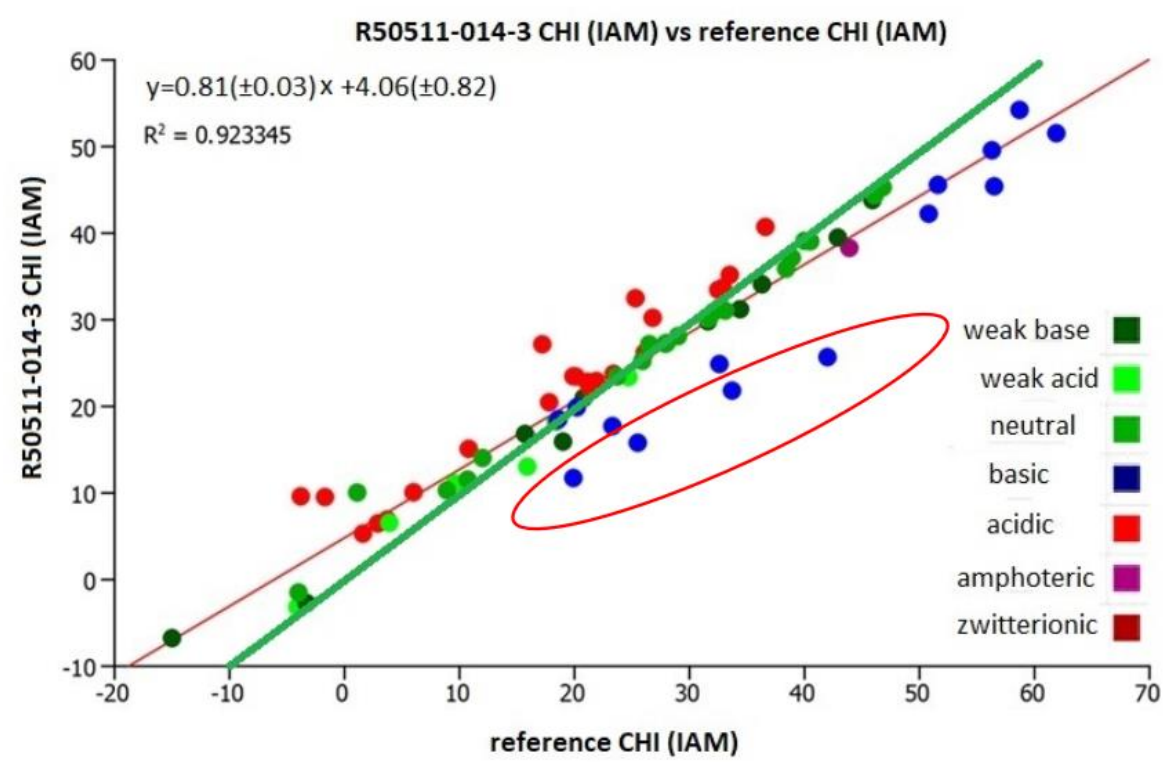

Figure 3. The plot of the $\mathrm{CHI}$ (IAM) values from the literature [13] (reference CHI (IAM)) with the CHI (IAM) values using IAM.PC.DD2 column R25011-014-3. The green line is the line of unity.

R20511-014-3 CHI (IAM) vs reference CHI (IAM)

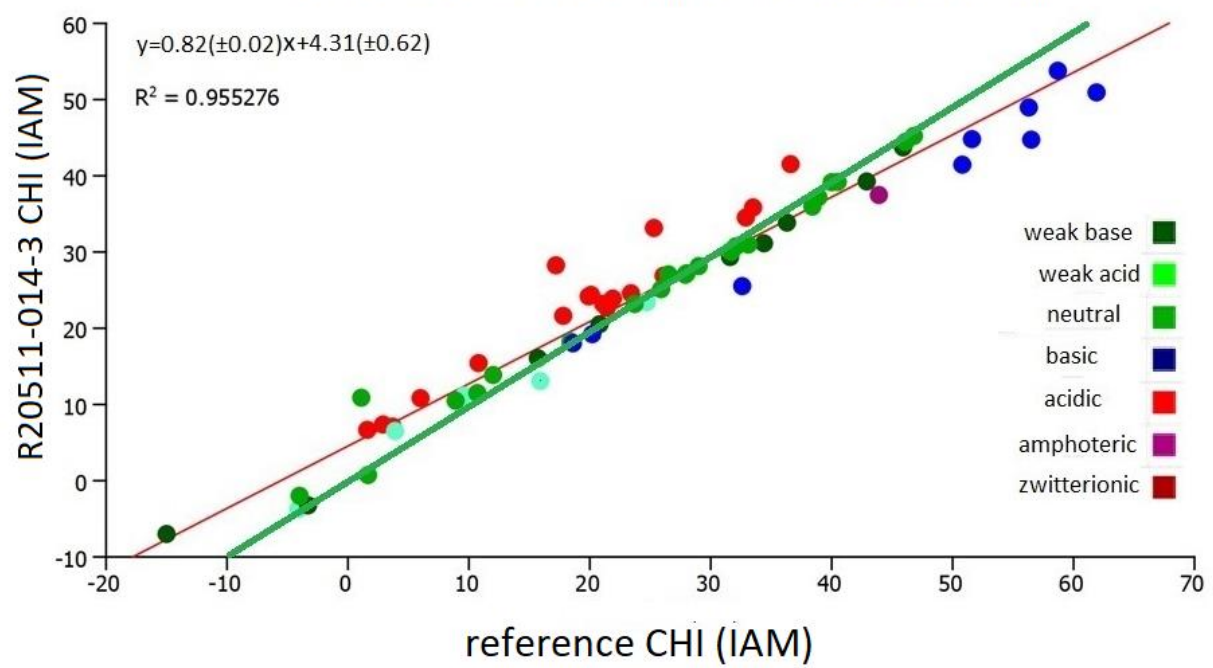

Figure 4. Comparison of the experimental CHI (IAM) values obtained on the IAM.PC.DD2 (R20511-014-3) column with the reference $\mathrm{CHI}$ (IAM) values without the 4 strong basic compounds. The green line is the line of unity 
The human clinical volume of distribution and protein binding data of the investigated marketed drugs

The volume of distribution model is based on two properties of the compounds, the IAM binding and the human serum albumin (HSA) binding. Therefore, the IAM binding data obtained using the new IAM.PC.DD2 columns were examined in the models of the human clinical volume of distribution together with the re-measured protein binding data.

Figure 5 shows the reproducibility of the HSA columns by plotting the newly measured log $K$ (HSA) values as a function of the "reference $\log K(\mathrm{HSA})$ " values published previously [13]. It can be seen that the slope is very close to 1 and the intercept is very close to 0 . There are slightly greater discrepancies between the reference and the re-measured $\log K(\mathrm{HSA})$ values for strongly bound compounds. This shows that the specific binding sites of the human serum albumin may vary only slightly from column to column. With this excellent agreement between the $\log K$ (HSA) values obtained on various Chiral HSA columns, it can be assumed that they would not affect the volume of distribution model which is based on two binding properties for the compounds, namely the IAM and HSA binding.

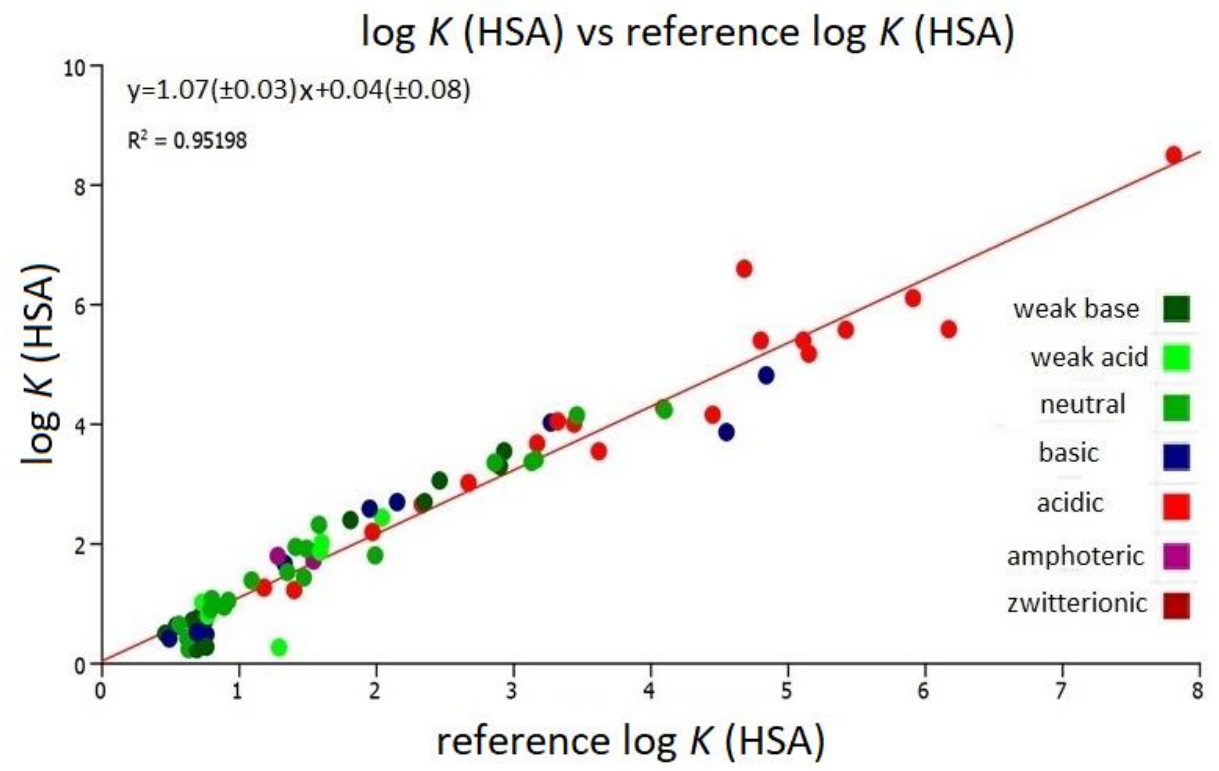

Figure 5 The plot of the log $K(\mathrm{HSA})$ values from the literature [13] and the re-measured log $K(\mathrm{HSA})$ for this study

Estimating the volume of distribution using the old and new IAM.PC.DD2 columns

The logarithmic values of the HSA and IAM binding data have been used to estimate the human clinical volume of distribution. Using the published equation (equation 4), the estimated values are compared using the IAM data obtained on columns R20511-014-3 and P20511-014-3 and the IAM data from the literature [12].

The estimated $\log V_{\text {dss }}$ values have been calculated using equation 4 and the measured $\log K(\mathrm{HSA})$ and $\log K$ (HSA) data obtained on the new IAM.PC.DD2 columns. The estimated log $V_{\text {dss }}$ values are listed in Table 5 together with the acid/base character of the compounds.

Figure 6 shows the plot of the estimated $\log V_{\text {dss }}$ values using the CHI (IAM) values obtained on the IAM.PC.DD2 column P20511-014-3 and those derived from the reference CHI (IAM) values. A good correlation was found between the estimated $\log V_{\text {dss }}$ values. In this study equation 4 was used with the same log $K(\mathrm{HSA})$ values and different log $K$ (IAM) values (depending on which IAM column was used). 
Table 5. The estimated $\log V_{\text {dss }}$ values of the investigated compounds using equation 4 and their acid/base character

\begin{tabular}{|c|c|c|c|c|}
\hline Drug name & Acid/base character & $\begin{array}{c}\mathrm{R} 20511-014-3 \\
\log V_{\mathrm{dss}} \\
\end{array}$ & $\begin{array}{c}\text { P20511-014-3 } \\
\log V_{\text {dss }}\end{array}$ & $\begin{array}{c}\text { reference } \\
\log V_{\text {dss }}\end{array}$ \\
\hline Acecainide & basic & -0.03 & -0.03 & 0.1 \\
\hline Acetanilide & neutral & -0.12 & -0.12 & -0.13 \\
\hline Acetazolamide & neutral & -0.47 & -0.47 & -0.46 \\
\hline Alclofenac & acidic & -1.04 & -1.02 & -1.1 \\
\hline Amoxapine & basic & 0.62 & 0.57 & 1.67 \\
\hline Bamethan & basic & 0.01 & 0 & 0.01 \\
\hline Betamethasone & neutral & 0.12 & 0.12 & 0.18 \\
\hline Carbamazepine & neutral & -0.07 & -0.07 & -0.09 \\
\hline Chlorpromazine & basic & 0.88 & 0.82 & 2.15 \\
\hline Cinoxacin & acidic & -0.38 & -0.37 & -0.42 \\
\hline Colchicine & neutral & 0.02 & 0.01 & 0.03 \\
\hline Cytarabine & weak base & -0.26 & -0.26 & -0.31 \\
\hline Diazoxide & weak acid & -0.19 & -0.19 & -0.16 \\
\hline Diclofenac & acidic & -0.6 & -0.57 & -0.67 \\
\hline Diprophylline & neutral & -0.22 & -0.23 & -0.24 \\
\hline Ethinyl estradiol & neutral & 0.28 & 0.27 & 0.39 \\
\hline Famotidine & weak base & -0.04 & -0.05 & -0.06 \\
\hline Felbamate & neutral & 0.07 & 0.07 & -0.64 \\
\hline Felodipine & neutral & 0.23 & 0.23 & 0.35 \\
\hline Fenoprofen & acidic & -1.02 & -0.98 & -1.27 \\
\hline Finasteride & neutral & 0.21 & 0.21 & 0.3 \\
\hline Floxacillin & acidic & -0.55 & -0.53 & -0.56 \\
\hline Flumazenil & neutral & -0.04 & -0.04 & -0.04 \\
\hline Flurbiprofen & acidic & -1.44 & -1.42 & -1.56 \\
\hline Furosemide & acidic & -0.7 & -0.68 & -0.71 \\
\hline Gemfibrozil & acidic & -1.31 & -1.27 & -1.34 \\
\hline Glipizide & acidic & -0.69 & -0.68 & -0.73 \\
\hline Griseofulvin & neutral & 0.04 & 0.03 & 0.12 \\
\hline Hydrochlorothiazide & weak acid & -0.18 & -0.17 & -0.13 \\
\hline Hydrocortisone & neutral & 0.1 & 0.09 & 0.12 \\
\hline Imipramine & basic & 0.66 & 0.6 & 1.16 \\
\hline Indomethacin & acidic & -0.72 & -0.69 & -0.96 \\
\hline Isradipine & neutral & 0.08 & 0.08 & 0.13 \\
\hline Ketoconazole & weak base & 0.12 & 0.1 & 0.32 \\
\hline Ketoprofen & acidic & -0.98 & -0.96 & -1.01 \\
\hline Labetalol & amphoteric & 0.38 & 0.34 & 0.71 \\
\hline Lignocaine & basic & 0.14 & 0.16 & 0.4 \\
\hline Methylprednisolone & neutral & 0.12 & 0.11 & 0.16 \\
\hline Metronidazole & weak base & -0.24 & -0.25 & -0.25 \\
\hline Minoxidil & weak base & -0.11 & -0.1 & -0.04 \\
\hline Nabumetone & neutral & -0.09 & -0.09 & 0.03 \\
\hline Nadolol & basic & -0.34 & -0.35 & -0.33 \\
\hline Nicardipine & weak base & 0.32 & 0.32 & 0.47 \\
\hline Nifedipine & neutral & -0.07 & -0.07 & -0.04 \\
\hline Nitrendipine & neutral & 0.08 & 0.08 & 0.16 \\
\hline
\end{tabular}




\begin{tabular}{lcccc}
\hline Papaverine & weak base & -0.13 & -0.13 & 0 \\
Pentoxifylline & neutral & -0.07 & -0.07 & -0.1 \\
Perphenazine & basic & 0.66 & 0.61 & 1.35 \\
Phenytoin & weak acid & -0.02 & -0.02 & 0.03 \\
Pindolol & basic & 0.14 & 0.13 & 0.85 \\
Prazosin & weak base & -0.11 & -0.13 & -0.05 \\
Prednisolone & neutral & 0.12 & 0.12 & 0.14 \\
Prednisone & neutral & 0.03 & 0.03 & 0.05 \\
Primidone & neutral & -0.13 & -0.13 & -0.15 \\
Probenecid & acidic & -0.66 & -0.64 & -0.75 \\
Procainamide & basic & -0.13 & -0.12 & 0.02 \\
Propanolol & basic & 0.64 & 0.59 & 1.29 \\
Propylthiouracil & weak acid & -0.31 & -0.31 & -0.34 \\
Proxyphylline & neutral & -0.75 & -0.74 & -0.85 \\
Sulfachlorpyridazine & acidic & -0.71 & -0.7 & -0.76 \\
Sulfameter & acidic & -0.57 & -0.57 & -0.61 \\
Sulfamethoxypyridazine & weak acid & -0.57 & -0.56 & -0.59 \\
Sulfinpyrazone & acidic & -0.64 & -0.62 & -0.65 \\
Sulfisoxazole & acidic & -0.68 & -0.67 & -0.72 \\
Sulphadimidine & amphoteric & -0.41 & -0.41 & 0.22 \\
Sulpiride & basic & -0.11 & -0.1 & 0.12 \\
Tamoxifen & basic & 0.94 & 0.89 & 1.48 \\
Theobromine & weak acid & -0.24 & -0.25 & -0.25 \\
Tolfenamic Acid & acidic & -0.54 & -0.49 & -0.76 \\
Trazodone & weak base & -0.09 & -0.1 & 0.01 \\
Trimethoprim & weak base & -0.05 & -0.06 & -0.06 \\
Warfarin & acidic & -0.69 & -0.68 & -0.78 \\
\hline & & & & \\
\hline
\end{tabular}

log Vdss P20511-014-3 vs reference log Vdss

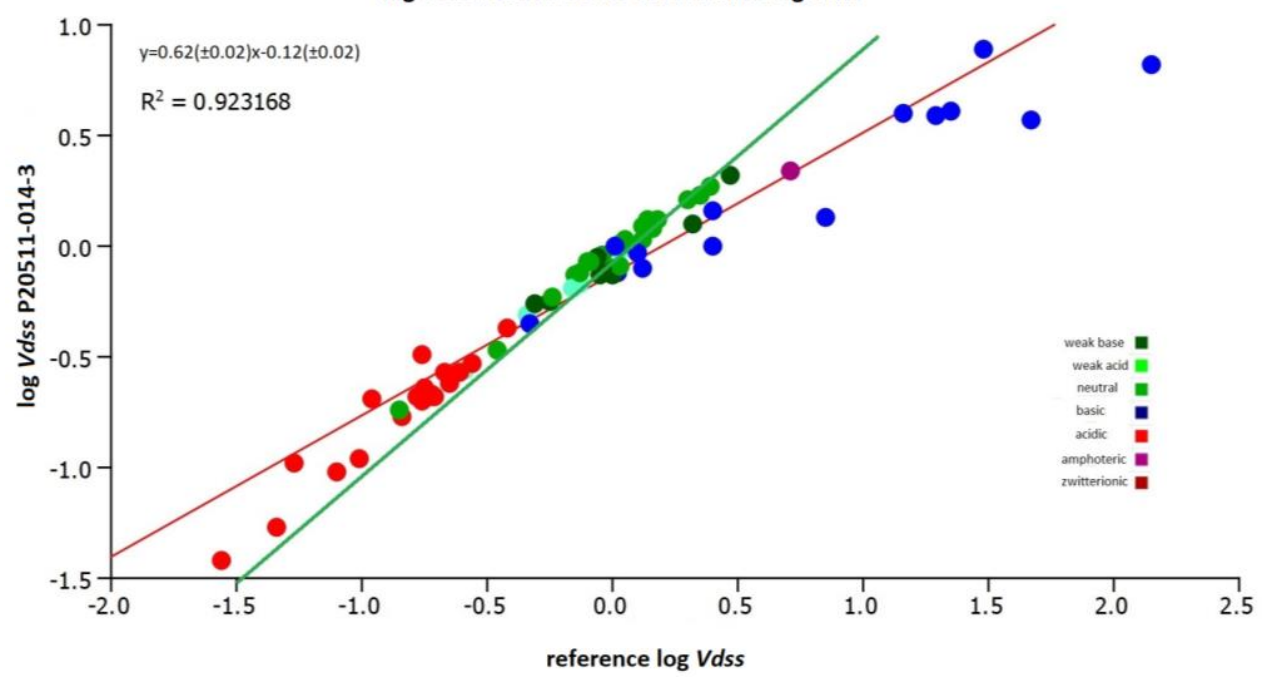

Figure 6. The plot of the estimated $\log V_{\text {dss }}$ values using the $\mathrm{CHI}$ (IAM) values obtained on the IAM.PC.DD2 column P20511-014-3 and from the reference [13]. The green line is the line of unity.

Figure 7 shows the plot of the estimated $\log V_{\text {dss }}$ values using the CHI (IAM) values obtained on the IAM.PD.DD2 column P20511-014-3 and those obtained from the reference but without the 4 strong basic compounds (procainamide, sulpiride, zolmitriptan and pindolol). It can be seen that the agreement is slightly improved with an $\mathrm{R}^{2}$ value of 0.96 , however, the slope is still less than one, while the intercept is 
close to zero. This means that the rank order of the volume of distribution data would be the same when using the new batches of IAM.PC.DD2 columns as it was when using the old batches of IAM columns. However, absolute values may be slightly different for the neutral, acidic and basic compounds and greater discrepancies can be expected for some strong basic compounds which are similar to those left out from the plot.

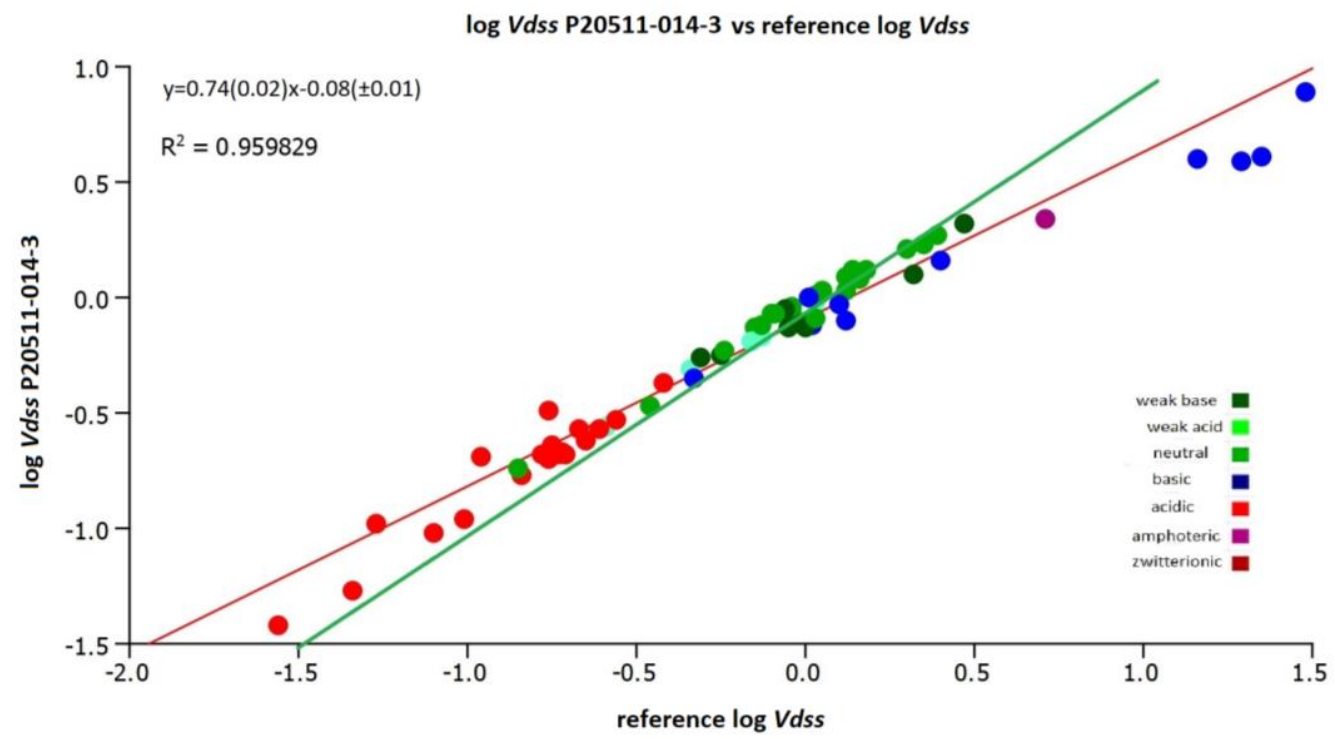

Figure 7. The plot of the estimated log $V_{\text {dss }}$ values using the CHI (IAM) values obtained on column P20511-0143 and from the reference values [12] without the four strong basic compounds. The green line is the line of unity.

It was important to investigate how the estimated volume of distribution using the $\mathrm{CHI}$ (IAM) data from the new batches of the IAM.PC.DD2 columns correlated with the actual clinical volume of distribution data. Figures 8 and 9 show the correlation of the estimated $\log V_{\text {dss }}$ values using the same coefficients as in equation 4 with the clinical $\log V_{\text {dss }}$ values. Figure 9 shows that the CHI (IAM) values obtained on the new batches of IAM.PC.DD2 column can be used to estimate the human clinical volume of distribution data by using equation 4 . There is better agreement with the clinical data when using the $\log V_{\text {dss }}$ obtained on the new batch of the IAM.PC.DD2 column. However, the absolute values may be slightly different and a correction factor (slope 0.62 ) will be required. In a drug discovery setting for ranking and differentiating compounds using an estimated $\log V_{\text {dss, }}$, these values can be used with confidence. However, it is important to acknowledge that certain strong basic compounds may appear to have a lower volume of distribution than expected.

It was also important to investigate how the $\mathrm{CHI}$ (IAM) and $\log K(I A M)$ values perform in other in vivo distribution models.

\section{Estimating brain tissue binding using the old and new batches of IAM.PC.DD2 columns}

The brain tissue binding can be estimated from the sum of the albumin and the phospholipid binding as described by equation 8 . The model was built on 135 drug discovery compounds from several neuroscience projects using rat brain tissue binding data obtained by equilibrium dialysis and measured HSA and IAM binding data as described in reference [12].

$\log k(\mathrm{BTB})=1.29( \pm 0.10) \log k(\mathrm{IAM})+1.03( \pm 0.10) \log k(\mathrm{HSA})-0.237$

$\mathrm{n}=135 \quad \mathrm{r}^{2}=0.76 \quad \mathrm{~s}=0.35 \quad \mathrm{~F}=212$

where $n$ is the number of compounds, $r^{2}$ is the correlation coefficient, $s$ is the standard error of the 
estimate and $F$ is the Fisher-test value.

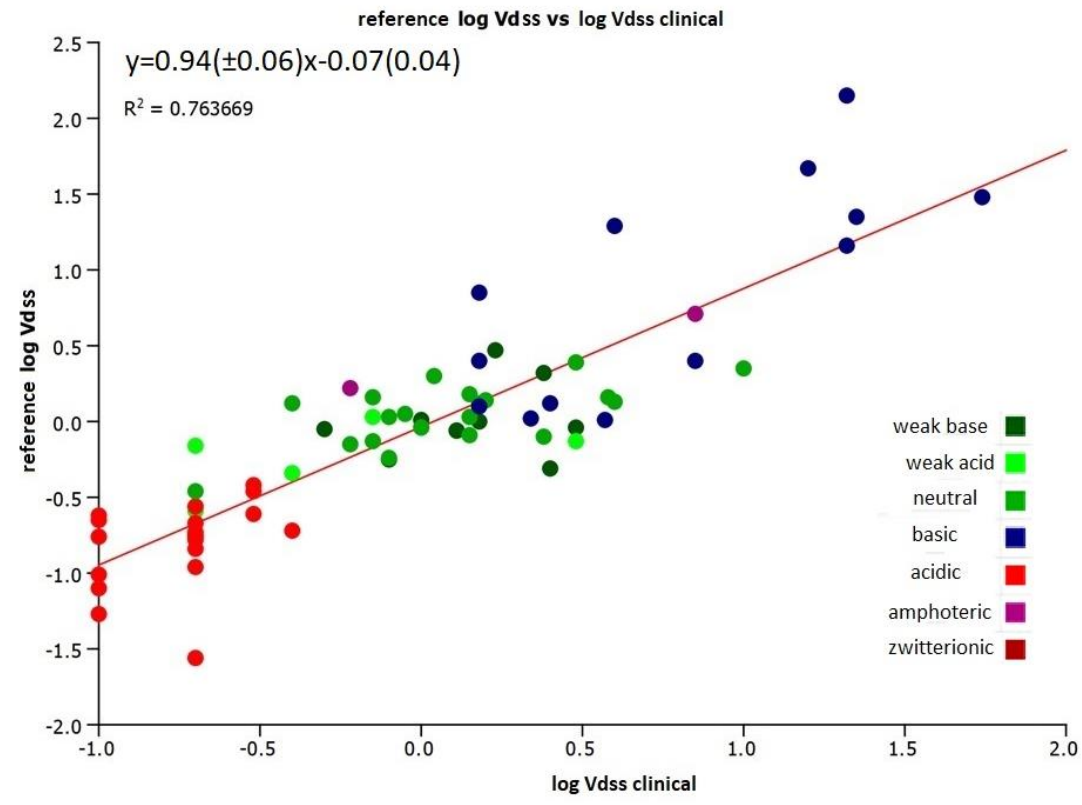

Figure 8. The plot of the estimated and the measured clinical volume of distribution data using the old batches of IAM columns (reference [13] reference $\log V_{\mathrm{dss}}$ ) using equation 4.

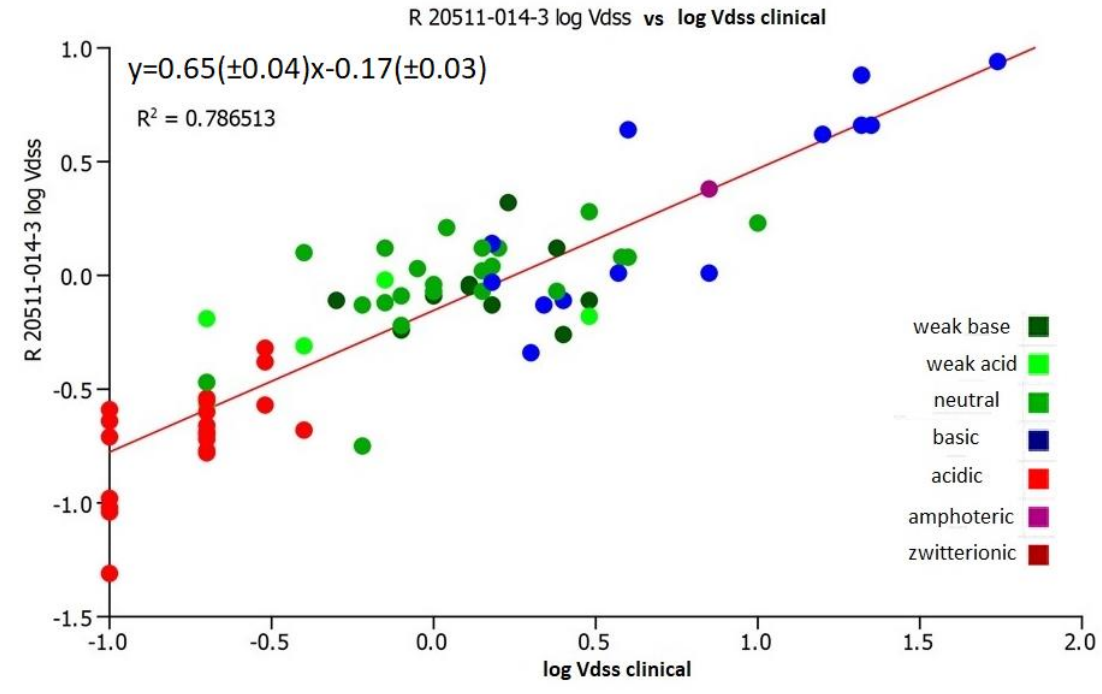

Figure 9. The plot of the estimated and measured clinical volumes of distribution using the new batch R20511-014-3 IAM.PC.DD2 column versus the reference $\log V_{\text {dss }}$ [13].

Figure 10 shows the comparison of the estimated brain tissue binding (\%BTB) when the old (reference [13]) and the new column P20511-014-3 IAM.PC.DD2 data was used in the model.

The agreement is very good with an $\mathrm{R}^{2}$ value of 0.95 , the slope is close to 1 and the intercept is close to zero using the CHI (IAM) values obtained on P20511-014-3 from the latest batch of IAM.PC.DD2 columns. However, the four basic compounds that showed significantly lower CHI (IAM) values are significant outliers resulting in these compounds being estimated to have lower brain tissue binding than their actual brain tissue binding.

Estimating the unbound volume of distribution using the old and new batches of IAM.PC.DD2 columns

The agreement between the estimated unbound volume of distribution data using the reference [13] CHI (IAM) values and the CHI IAM values obtained on column R25011-014-3 are shown in Figure 11. 


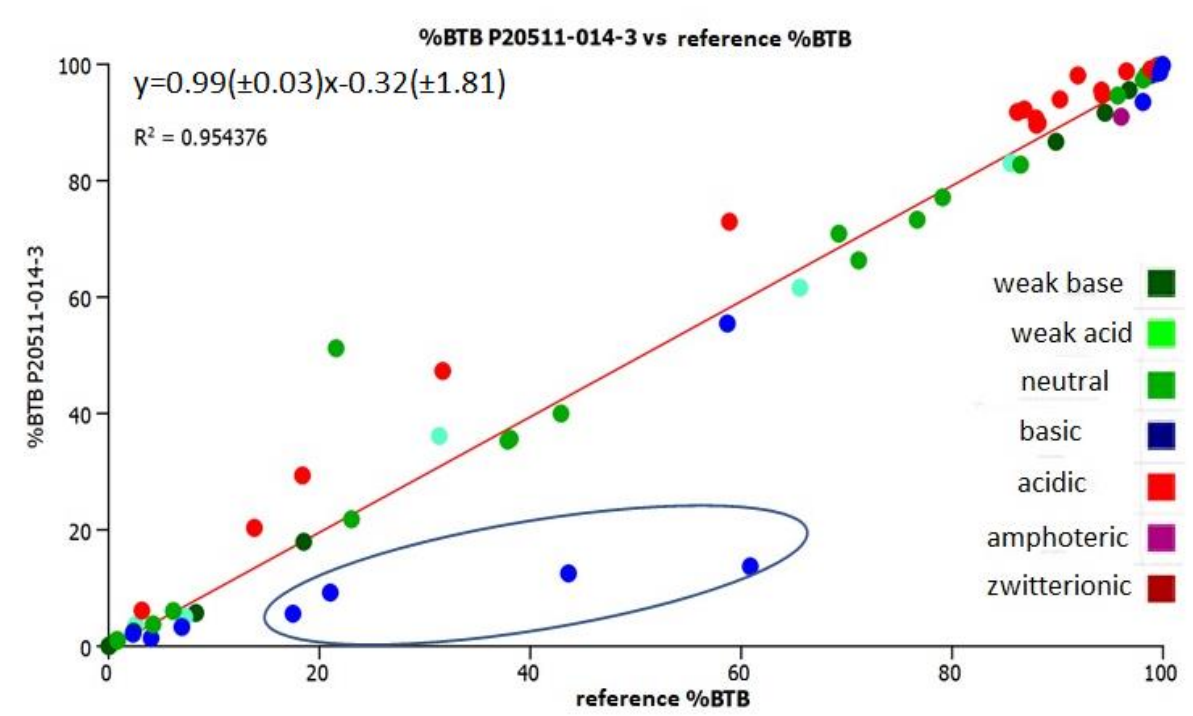

Figure 10. The plot of the estimated brain tissue binding (\%BTB) obtained using the IAM data obtained from the reference [13] and that obtained using IAM.PC.DD2 column P20511-014-3

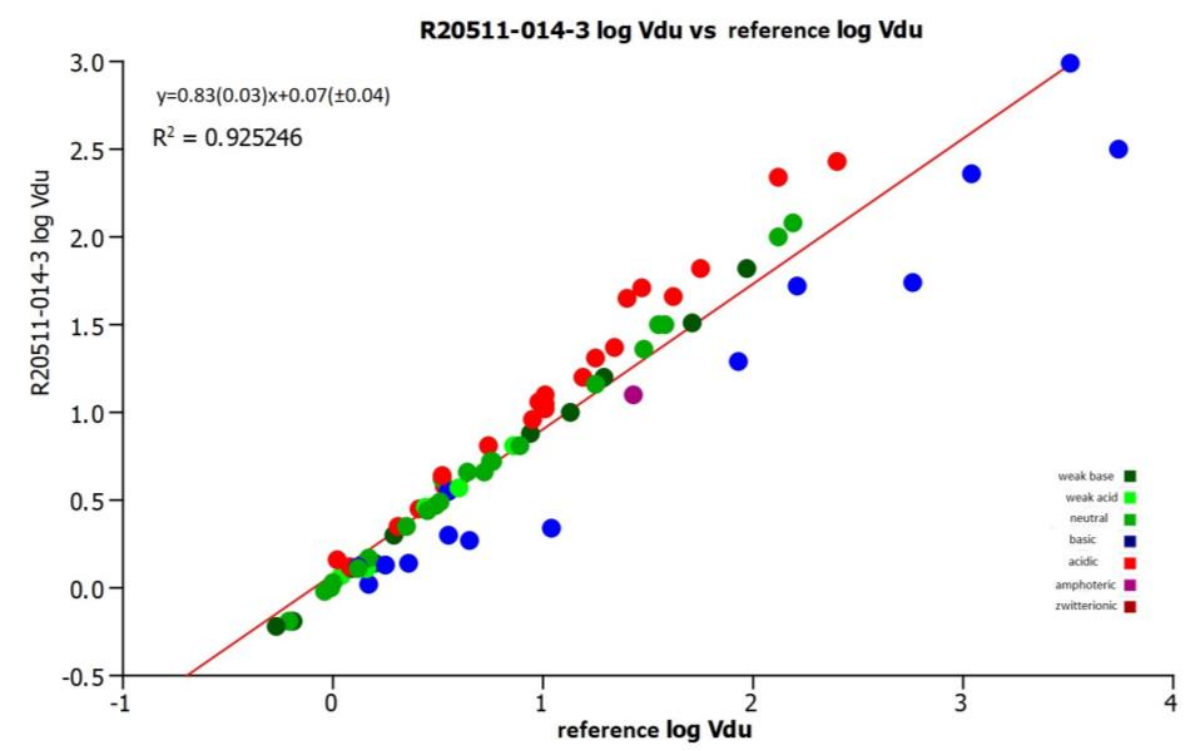

Figure 11. A plot of the estimated unbound volume of distribution using the IAM data from the reference (reference [12]) and on the IAM.PC.DD2 R20511-014-3 column

It can be seen that the estimated unbound volume of distribution using the $\mathrm{CHI}$ (IAM) values obtained on column R20511-014-3 shows good agreement with the logVdu from the reference CHI (IAM) values. It can be observed from the plot that basic compounds tend to show a lower unbound volume of distribution using the CHI (IAM) values obtained on the new column R20511-014-3.

\section{Estimating drug efficiency using the old and new batches of IAM.PC.DD2 columns}

Figure 12 shows the comparison of the estimated drug efficiency values using the old and a new batch (R20511-014-3) of IAM.PC.DD2 column.

There is good agreement between the drug efficiency values obtained on the new batch of the IAM column and the literature data. However, the basic compounds showed greater drug efficiency than expected when using the new batch (R20511-014-3) of IAM.PC.DD2 column. 


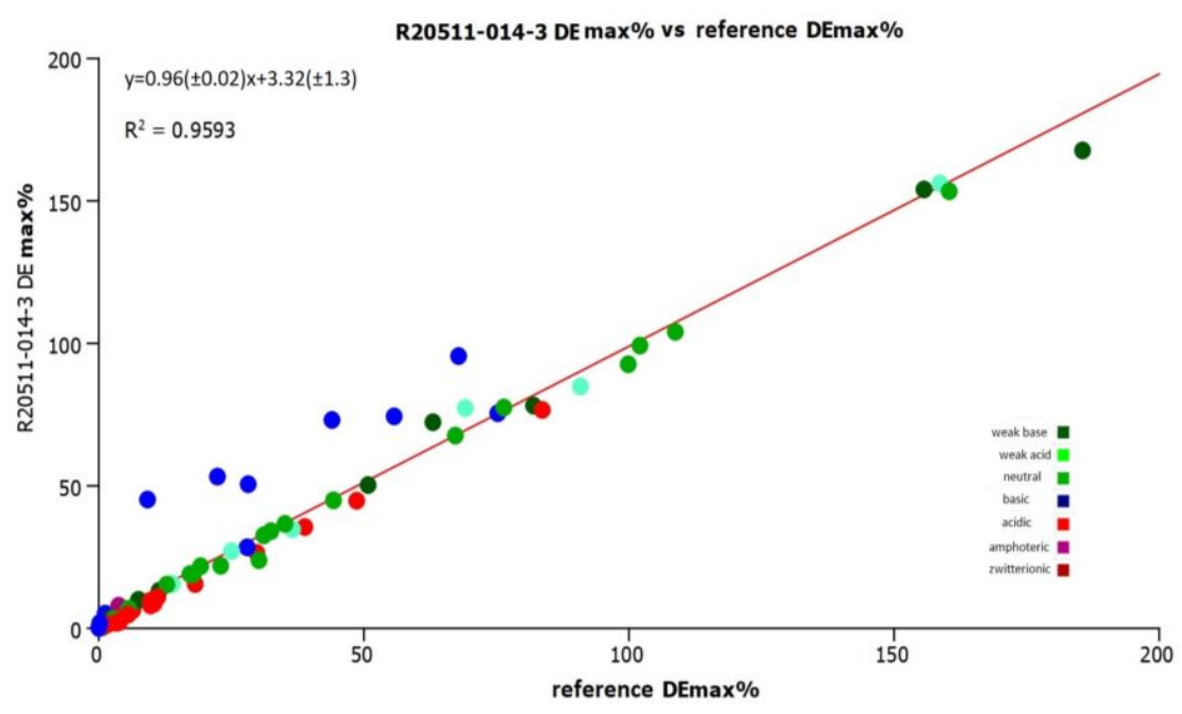

Figure 12. The plot of the drug efficiency values for the investigated drugs using the IAM data obtained from the reference (reference [12]) and by R20511-014-3 column

In conclusion, some differences between the old and the latest batches of IAM.PC.DD2 columns were observed when estimating the in vivo distribution of compounds. The discrepancies are more significant when the models are based on the differences between the measured IAM and HSA binding (volume of distribution). When the models are based on the sum of the two types of binding, the correlations between the estimated values (brain tissue binding, the unbound volume of distribution and drug efficiency) are much stronger. However, systematic differences could be observed for basic compounds in the estimated drug efficiency values. Polar basic ( $\log P$ less than 2 ) compounds with over 6 rotatable bonds were estimated to have greater dug efficiency values than previously estimated using the old batches of IAM.PC.DD2 columns. These discrepancies are due to the weaker interaction of the new batches of IAM.PC.DD2 stationary phases with positively charged basic compounds. This could be the result of better endcapping procedures to block the free silanol groups and it could also be due to the higher purity of the silica providing less overall negatively charged silanols.

Based on these observations it was decided to investigate the potential benefit of developing new models using the data obtained on the new silica based IAM stationary phase and to compare the new models with the existing model equation (equation 4).

\section{Developing new distribution models using the new batches of IAM.PC.DD2 columns}

The correlations between the old and the new batches of IAM retention data have been investigated. It was found that the reference $\mathrm{CHI}$ (IAM) data can be estimated using the $\mathrm{CHI}$ (IAM) data obtained on the new batches (P20511-014-3 and R20511-014-3) of IAM.PC.DD2 columns when the retention difference on C-18 columns obtained at $\mathrm{pH} 10.6$ and $\mathrm{pH} 2.6$ are included in the equation (equation (9)). This second variable significantly increased the explained variance of the reference $\mathrm{CHI}$ (IAM).

Reference $\mathrm{CHI}(\mathrm{IAM})=1.06( \pm 0.03) \mathrm{CHI}(\mathrm{IAM})(\mathrm{R}-20511-01403)+0.008( \pm 0.01) \Delta \mathrm{CHI}(\mathrm{pH} 10.5-\mathrm{pH} 2.6)-1.06$ $( \pm 0.73)$

$$
\mathrm{n}=67 \quad \mathrm{r}^{2}=0.97 \mathrm{~s}=2.7 \quad \mathrm{~F}=1100
$$

The correlation was slightly improved (from 0.92 to 0.97 ) between the reference $\mathrm{CHI}$ (IAM) values and the $\mathrm{CHI}$ (IAM) values obtained on the new batch of IAM.PC.DD2 columns. However, in order to be able to use equation 9, the $\mathrm{CHI}$ measurements on $\mathrm{C}-18$ columns should be carried out at high and low $\mathrm{pH}$. This 
requires two additional measurements for each compound. When resources are limited, the calculated molecular descriptor can be used. The following parameters have been calculated for the investigated drug molecules: $\log \mathrm{P}$, molecular weight, number of $\mathrm{H}$-bond donor and acceptor groups, total polar surface area, flexibility and the number of rotatable bonds (NRB). It was found that the only significant variables among the calculated parameters that improved the correlation between the $\mathrm{CHI}$ (IAM) values obtained using the new and old batches of IAM.PC.DD2 columns were clogP and the total polar surface area (TPSA) as shown in equation 10. However, the correlation coefficient improved only slightly from 0.92 to 0.96 .

Reference CHI $($ IAM $)=1.38( \pm 0.07) \mathrm{CHI}(\mathrm{IAM})(\mathrm{R} 20511-014-3)-2.42( \pm 0.57) \operatorname{clog} \mathrm{P}-0.03( \pm 0.01)$ TPSA -1.28

$$
\mathrm{n}=68 \quad \mathrm{r}^{2}=0.96 \quad \mathrm{~s}=3.3 \quad \mathrm{~F}=530
$$

The other option was to build a new model for the estimation of the volume of distribution using the new batches of IAM.PC.DD2 columns and the clinical volume of distribution data.

The new equation for modelling clinical steady-state volume of distribution data is shown in equation 11. The mathematical-statistical parameters are very similar to the original equation (Equation 4); however, the coefficients of the IAM and HSA binding data are very different.

$$
\begin{aligned}
& \log V_{\text {dss }} \text { clinical }=0.54( \pm 0.04) \log K(\text { IAM })(\text { R20511-01403 })-0.24( \pm 0.02) \log K(\text { HSA })-0.7 \\
& n=72 \quad r^{2}=0.78 \quad s=0.30
\end{aligned}
$$

The plot of the estimated and measured clinical steady-state volume of distribution data are shown in Figure 13. When comparing the coefficients of equation 4 (the published $V_{\text {dss }}$ model equation) and equation 10, it can be seen that the coefficients for the HSA binding do not differ significantly ( 0.24 and 0.22 with an error of 0.02 ). However, the coefficients of $\log K(I A M)$ are different at the $95 \%$ confidence interval and agree only within a $90 \%$ confidence interval: $0.54 \pm 0.04$ and $0.44 \pm 0.02$.

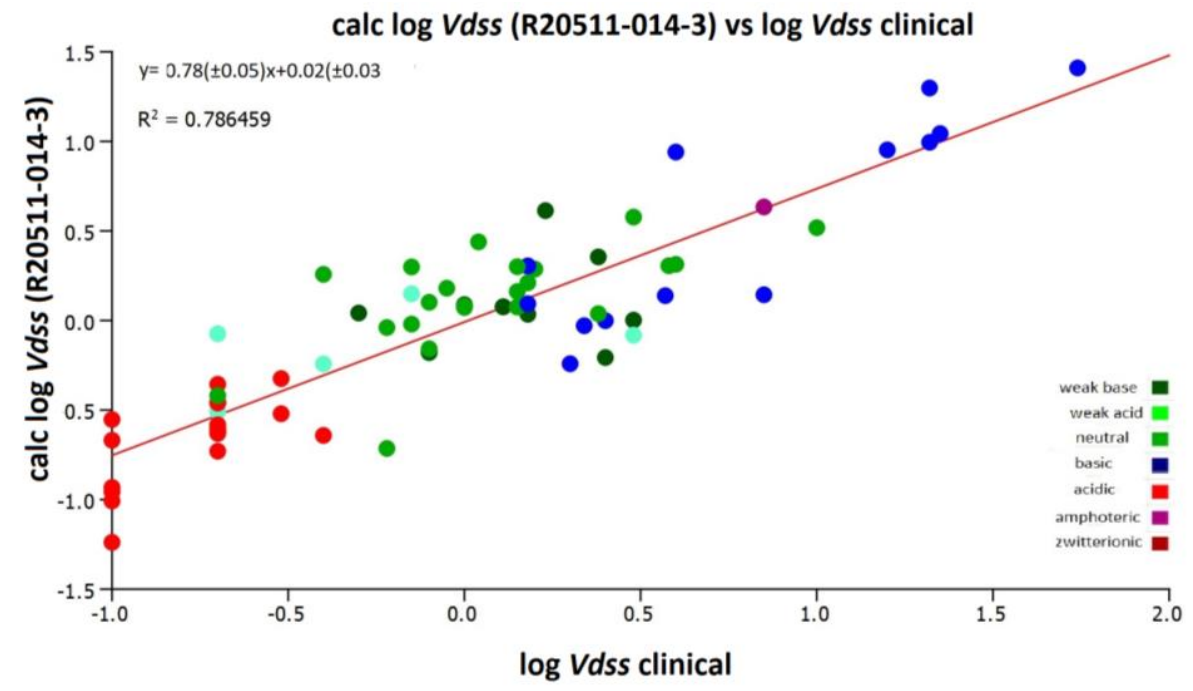

Figure 13. The plot of the calculated $\log V_{\mathrm{dss}}$ values using the new model with the CHI (IAM) values obtained on column (R20511-014-3) as a function of the clinical $\log V_{\text {dss }}$ values.

\section{Conclusions}

The use of a new silica support provided a slightly different selectivity for the new IAM.PC.DD2 columns regardless of the bonding procedure used. Therefore, new $\mathrm{CHI}$ (IAM) values have been proposed for the system suitability test compounds. New IAM.PC.DD2 columns should be evaluated using the new CHI (IAM) values (Table 4.). The reproducibility of the new batches of IAM.PC.DD2 columns are very good and are 
within $2 \mathrm{CHI}$ (IAM) units. The effect of the selectivity change has been investigated on previously developed in vivo models for drug distribution that are based on $\mathrm{CHI}$ (IAM) values. A set of 72 marketed drugs with clinical distribution data (volume of distribution, unbound volume of distribution and drug efficiency) have been analyzed on the new batches of IAM columns. The CHI (IAM) values were compared with the values obtained from the previous batches of IAM columns and it was observed that small polar basic compounds showed significantly shorter retention times on the new IAM.PC.DD2 columns. The correlation coefficient improved significantly when the data from these compounds are removed from the analysis. The possible reason for these discrepancies may be due to the better endcapping procedure on the new IAM columns which is blocking the silanol effects that increase retention of basic compounds. It is also likely that the newer "Type B" silica is higher purity silica with a more inert surface and creates fewer negatively charged silanols decreasing the amount of non-specific binding. It was found that using the new CHI (IAM) data in the previously published model equations, a good correlation was obtained with the original values. However, the absolute values were different but the rank order for the in vivo distribution values remain the same using the old and the new columns. New models have also been developed for estimating the clinical volume of distribution of compounds using the data from the new IAM columns. The mathematical statistical characteristics of the new models are similar, but the coefficients of the CHI (IAM) and $\log K$ (HSA) parameters are slightly different. Since the correlation between the estimated and clinical volume of distribution data was the same or slightly better with the data obtained from the new batches of IAM columns, it is expected that the in vivo estimates can be as accurate with the data obtained from the new batches of IAM columns as with the older batches of IAM columns. The newly derived equation (eqation 11) for the estimation of the volume of distribution is suggested when someone begins to use biomimetic chromatographic measurements with the new columns. However, reference can still be made to the existing data previously published using the older columns.

Conflict of interest: Klara Valko is the founder of Bio-Mimetic Chromatography Ltd. Scott Anderson is director at Regis Technologis inc, while Shenaz Bunally is scientist at Physicochemical group at GSK.

\section{References}

[1] C. Pidgeon, U.V. Venkataram. Immobilized artificial membrane chromatography: Supports composed of membrane lipids. Anal. Biochem. 176 (1989) 36-47.

[2] K. Valkó, P. Slégel. New chromatographic hydrophobicity index $(\phi 0)$ based on the slope and the intercept of the log k' versus organic phase concentration plot. J. Chromatogr. A, 631 (1993) 49-61.

[3] K. Valko, C.M. Du, C.D. Bevan, D.P. Reynolds, M.H. Abraham. Rapid-gradient HPLC method for measuring drug interactions with immobilized artificial membrane: Comparison with other lipophilicity measures. J. Pharm. Sci. 89 (2000) 1085-1096.

[4] K. Valko, S. Teague, C. Pidgeon. In vitro membrane binding and protein binding (IAM MB/PB technology) to estimate in vivo distribution: Applications in early drug discovery. ADMET DMPK 5 (2017) 14-38.

[5] Z. Jiang, J. Reilly. Chromatography approaches for early screening of the phospholipidosis-inducing potential of pharmaceuticals. J. Pharm. Biomed. Anal. 61 (2012) 184-190.

[6] F. Tsopelas, C. Giaginis, A. Tsantili-Kakoulidou. Lipophilicity and biomimetic properties to support drug discovery. Expert Opin. Drug Discov. 12 (2017) 885-896.

[7] F. Tsopelas, T. Vallianatou, A. Tsantili-Kakoulidou. Advances in immobilized artificial membrane (IAM) chromatography for novel drug discovery. Expert Opin. Drug Discov. 11 (2016) 473-478.

[8] S. Bunally, R.J. Young. The role and impact of high throughput biomimetic measurements in drug discovery, ADMET and DMPK 6 (2018) 74-84. 
[9] A. Taillardat-Bertschinger, C.A. Martinet, P.A. Carrupt, M. Reist, G. Caron, R. Fruttero, B. Testa. Molecular Factors Influencing Retention on Immobilized Artifical Membranes (IAM) Compared to Partitioning in Liposomes and n-Octanol. Pharm. Res. 19 (2002) 729-737.

[10] E. Lázaro, C. Ràfols, M.H. Abraham, M. Rosés. Chromatographic estimation of drug disposition properties by means of immobilized artificial membranes (IAM) and C18 columns. J. Med. Chem. 49 (2006) 4861-4870.

[11] F. Barbato, G. di Martino, L. Grumetto, M.I. La Rotonda. Prediction of drug-membrane interactions by IAM-HPLC: effects of different phospholipid stationary phases on the partition of bases. Eur. J. Pharm. Sci. 22 (2004) 261-269.

[12] L. Grumetto, G. Russo, F. Barbato. Immobilized Artificial Membrane HPLC Derived Parameters vs PAMPA-BBB Data in Estimating in Situ Measured Blood-Brain Barrier Permeation of Drugs. Mol. Pharm. 13 (2016) 2808-2816.

[13] F. Hollosy, K. Valko, A. Hersey, S. Nunhuck, G. Keri, C. Bevan. Estimation of Volume of Distribution in Humans from HPLC Measurements of Human Serum Albumin Binding and Immobilized Artificial Membrane Partitioning. J. Med. Chem. 49 (2006) 6958-6971.

[14] K. Valko. Physicochemical and Biomimetic Properties in Drug Discovery: Chromatographic Techniques for Lead Optimization. Wiley Hoboken NJ, 2014.

[15] F. Tsopelas, T. Vallianatou, A. Tsantili-Kakoulidou. The potential of immobilized artificial membrane chromatography to predict human oral absorption. Eur. J. Pharm. Sci. 81 (2016) 82-93.

[16] X. Sui, J. Sun, H. Li, Y. Wang, J. Liu, X. Liu, W. Zhang, L. Chen, Z. He. Prediction of volume of distribution values in human using immobilized artificial membrane partitioning coefficients, the fraction of compound ionized and plasma protein binding data. Eur. J. Med. Chem. 44 (2009) 44554460.

[17] K.L. Valkó, S.B. Nunhuck, A.P. Hill. Estimating Unbound Volume of Distribution and Tissue Binding by in vitro HPLC-based Human Serum Albumin and Immobilized Artificial Artificial MembraneBinding Measurements. J. Pharm. Sci. 100 (2011) 849-862.

[18] K. Valko, E. Chiarparin, S. Nunhuck, D. Montanari. In vitro measurement of drug efficiency index to aid early lead optimization. J. Pharm. Sci. 101 (2012) 4155-4169.

[19] S. Teague, K. Valko. How to identify and eliminate compounds with a risk of high clinical dose during the early phase of lead optimisation in drug discovery. Eur. J. Pharm. Sci. 110 (2017) 37-50.

[20] A. Casartelli, M. Bonato, P. Cristofori, F. Crivellente, G. Dal Negro, I. Masotto, C. Mutinelli, K. Valko, V. Bonfante. A cell-based approach for the early assessment of the phospholipidogenic potential in pharmaceutical research and drug development. Cell Biol. Toxicol. 19 (2003) 161-176.

[21] E. Lounkine, M.J. Keiser, S. Whitebread, D. Mikhailov, J. Hamon, J. Jenkins, P. Lavan, E. Weber, A.K. Doak, S. Côté, B.K. Shoichet, L. Urban. Large Scale Prediction and Testing of Drug Activity on SideEffect Targets," Nature, 486 (2012) 361-367.

[22] F. Hollosy, K. Valko, A. Hersey, S. Nunhuck, G. Keri, C. Bevan. Estimation of Volume of Distribution in Humans from HPLC Measurements of Human Serum Albumin Binding and Immobilized Artificial Membrane Partitioning. J. Med. Chem. 49 (2006) 6958-6971.

[23] K. Valko, S. Nunhuck, C. Bevan, M. Abraham, D. Reynolds. Fast gradient HPLC method to determine compounds binding to human serum albumin. Relationship wth octanol water and immobilized artificial membrane lipophilicity. J. Pharm. Sci. 92 (2003) 2236-2248. 